\title{
Continuing Education
}

\section{Pharmacoepidemiology: The essentials}

\author{
Saket J. Thaker, Nithya J. Gogtay, Urmila M. Thatte* \\ Department of Clinical Pharmacology, Seth GS Medical College and KEM Hospital, Parel, Mumbai, \\ Maharashtra 400 012, India
}

\section{A R T I C L E I N F O}

Article history:

Received 11 January 2014

Accepted 18 April 2014

Available online 20 May 2014

Keywords:

Pharmacoepidemiology

Pharmacovigilance

Study designs

Databases

\begin{abstract}
A B S T R A C T
Aim: It is useful for a clinician to have a working knowledge of this science and hence this article reviews the essentials of pharmacoepidemiology for the practicing clinician.

Methods: This article is a compilation of information and examples from textbooks, guidelines, and various published articles and some real life examples to make it understandable.

Results: This article discusses various types of pharmacoepidemiology study designs, their merits/demerits, growing importance of databases in research and methodological issues involved in pharmacoepidemiology.

Conclusion: This article will sensitize clinicians regarding the importance of pharmacoepidemiology in their routine practice.
\end{abstract}

Copyright $\odot$ 2015, INDIACLEN. Publishing Services by Reed Elsevier India Pvt Ltd. All rights

reserved.
"A desire to take medicine is, perhaps, the great feature, which distinguishes man from other animals."

Sir William Osler, 1891

Perhaps it is this desire (or need) to take medicines that led to the development of the science of clinical pharmacology, which studies the use of medicines in man. As the population, complexity of diseases and the medicines developed to manage these pathologies burgeoned, the branch of pharmacoepidemiology (PE) - the study of the use of and the effects of drugs in large numbers of people evolved.

Traditionally, this branch developed because of the increasing need to generate data on the safety of a medicine after it had been marketed. During the clinical development (preclinical studies, phase I, II and phase III studies) of a drug, its efficacy and safety is evaluated under tightly controlled conditions in a relatively small number of people. ${ }^{1}$ Thus, a large amount of information about the safety of a drug really emerges only in the post-marketing phase, as more and more individuals are exposed to the drug. Additionally, once a product is marketed, there is not only a large increase in the number of patients exposed to the molecule, but it is now patients having co-morbid conditions and those being treated with concomitant medical products that receive the medicine. Therefore, conducting post-marketing PE studies generate very crucial data that allows for an ongoing risk assessment and characterization of a product's risk profile. Further, informed decisions on risk minimization can be made., ${ }^{2,3}$ Today almost $3-4 \%$ of drugs are withdrawn after marketing due to altered safety data that emerges through pharmacoepidemiology studies. ${ }^{4}$ Typical examples of PE data supporting

\footnotetext{
* Corresponding author. Tel.: +91 22 24174420, +91 22 24133767; fax: +91 2224112871.

E-mail address: urmilathatte@kem.edu (U.M. Thatte). 
drug withdrawals post-marketing include thalidomide, rimonabant and rosiglitazone. ${ }^{5-7}$

Similarly, in the clinical development phase, it is not possible to assess the "effectiveness" (as against efficacy which is measured in randomized controlled trials) of a drug in "the real world", as well as its place in the health care system. ${ }^{4,8}$ The science of Pharmacoepidemiology helps in generation of this type of data.

Pharmacoepidemiology (PE) comprises of two words 'pharmaco' and 'epidemiology' and encompasses aspects of both clinical pharmacology and epidemiology. Formally, "PE is the study of the use and effects/side-effects of drugs in large numbers of people with the purpose of supporting rational and cost-effective drug use in the population thereby improving health outcomes. ${ }^{9}$ The data obtained from PE studies guide decisions for national/international guideline generation, at health care facility level, as well as the individual patient level. Pharmacoepidemiology deals with several facts of drug research, including the utilization, benefits, and risks of drugs. It is useful for a clinician to have a working knowledge of this science and hence this article reviews the essentials of pharmacoepidemiology for the practicing clinician.

\section{Study designs for pharmacoepidemiological studies}

As in all clinical research study designs, PE studies are broadly of two types, namely experimental and observational studies. ${ }^{10,11}$ While experimental studies randomize patients to either the treatment or the control group, observational studies observe patients either on or not on the treatment of interest to find out the association between exposure and disease. ${ }^{12}$

When an exposure and outcome is to be studied in special cases and it is unethical to do randomization, observational studies are more suitable, e.g. effect of a drug in pregnant women or to prove whether exposure to a drug leads to cancer. Observational studies are further classified into descriptive and analytical studies. Analysis of secular trends is an example of descriptive observational studies while cross-sectional, cohort and case-control studies are classified as analytical observational studies. ${ }^{12}$ Observational studies are operationally simple, shorter in time duration and less costly as compared to experimental studies. Observational studies are useful to prove the effectiveness of drugs at times, for example, the pivotal study conducted on 30,569 patients in Canada that assessed the effect of corticosteroids in asthma, which showed $21 \%$ less mortality in the patients who were given corticosteroids. ${ }^{13}$

\section{Case reports/series}

While a case report is an event seen in a single patient, a case series describes all the patients who had the same exposure and had a similar outcome. ${ }^{10}$ Although it is data only from one (in a case report) or a few (in a case series) patients, and cannot provide a causal association between exposure and the event with any degree of certainty, often such case reports/series can help generate a hypothesis which can then be put to test with rigorous designs in a larger population. These are epidemiological tools used for the purpose. The case series describing valvular heart disease in 24 young (otherwise healthy) women (along with pulmonary hypertension in 8 of them) while taking Phentermine-Fenfluramine combination for weight reduction changed the medical practice instantaneously and also led to regulatory decisions. ${ }^{14}$

\section{Observational study designs}

\subsection{Analysis of secular trends}

"Analyses of secular trends" examine trends in disease events over time or across different geographical locations and correlate them with trends in putative exposures, such as rates of drug utilization. The unit of observation is a subgroup of a population rather than individuals. These are also called "ecological studies", e.g. a study of the sale of NSAIDs over a particular period of time and death due to major bleeding. ${ }^{4}$

\subsection{Case control studies}

Case control studies, are retrospective in nature, and are the common first approach to test causal hypothesis. The case control study has following distinct features: a). Both exposure and outcome should have occurred before the start of the study b). The study proceeds from effect to cause and c). Control group is always needed to support or refute an inference. ${ }^{12}$ Case control studies compare cases with controls with respect to exposure and are useful to study multiple outcomes of a single disease. They are easier to perform and logistically more feasible. ${ }^{15}$ Case control studies do not give direct association between the exposure and outcome, rather only an "odds ratio" [An odds ratio (OR) is a measure of association between an exposure and an outcome]. ${ }^{16}$ That is, at the end of the study the odds of getting the outcome with the exposure can be predicted. For example, Weil J et al (2000) ${ }^{17}$ studied the records of 1121 patients hospitalized for bleeding peptic ulcer and 989 controls from the population to study the risk factors for this condition with a focus on the association with the use of drugs. They found the adjusted (adjusted for the multiple exposures that would influence the same outcome) odds ratio and $95 \%$ confidence interval (CI) of having peptic ulcer bleeding were 3.6 [2.9-4.5] for NSAIDs, 2.7 [1.3, 4.5] for corticosteroids, 7.8 [2.8, 21.5] for warfarin. Additionally, they also found a significant interaction (effect modification) between simultaneous NSAIDs and corticosteroids use (Odds ratio; $9.0[4.9,10.2]) .{ }^{17}$

\subsection{Cohort studies}

A cohort consists of a group of people with the same characteristics, having had the same "exposure" (in the case of PE studies, this is usually a drug) that is followed over time to determine the occurrence of an outcome. ${ }^{12}$ The comparator group may be the general population from which the cohort is drawn, or it may be another cohort of persons thought to have had little or no exposure to the drug under investigation, but otherwise similar. ${ }^{15}$ Alternatively, subgroups within the cohort may be compared with each other. Cohort studies could be prospective or retrospective. ${ }^{18}$ In contrast to case control studies 
(which, as we saw earlier, predict the Odds Ratio) cohort studies give Relative Risk and Attributable Risk. The Relative Risk is the ratio of the probability of the outcome occurring (for example, developing a disease or being injured) in an exposed group to the probability of the outcome occurring in a comparable group not exposed to the drug. Attributable risk is the difference in the rate of occurrence of the outcome in an exposed population and an unexposed population and indicates to what extent the disease under study can be attributed to the exposure.

As against case-control studies, in cohort studies, multiple and rare outcomes can be studied. However, cohort studies are lengthy, operationally more difficult and costly. A classic cohort study was the "The Royal College of General Practitioners Oral Contraception Study" which followed 46,000 women who were using oral contraceptive (OC) and an equal number of women who were not taking OCs chosen from the same British general practices for 25 years. They found that for the entire period of follow-up the risk of death from all causes was similar in ever users and never users of oral contraceptives (relative risk $=1.0,95 \%$ confidence interval [0.9, 1.1]) and the risk of death for most specific causes did not differ significantly in the two groups. However, among current and recent (within 10 years) users the relative risk of death from ovarian cancer was 0.2 (95\% CI $[0.1,0.8])$, from cervical cancer 2.5 (95\% CI $[1.1,6.1])$, and from cerebrovascular disease 1.9 (95\% CI [1.2, 3.1]). By contrast, for women who had stopped use $\geq 10$ years previously there were no significant excesses or deficits either overall or for any specific cause of death. ${ }^{19,20}$ Stampfer MJ et al (1991) ${ }^{21}$ followed 48,470 postmenopausal women, who had previously participated in the Nurses' Health Study, aged between 30 and 63 years, and who did not have a history of cancer or cardiovascular disease at base line. During up to 10 years of follow-up (337, 854 person-years), they found incidents of 224 strokes, 405 cases of major coronary disease (nonfatal myocardial infarctions or deaths from coronary causes), and 1263 deaths from all causes. They found that after adjustment for age and other risk factors, the overall relative risk of major coronary disease in women currently taking estrogen was 0.56 (95\% CI $[0.40,0.80])$ and the risk was significantly reduced among women with either natural or surgical menopause. ${ }^{21}$ These examples illustrate how PE studies assist in forming evidence-based drug use guidelines.

\section{Interventional study designs}

\subsection{Randomized controlled trials in pharmacoepidemiology}

The randomized controlled trial (RCT) is the gold standard used to demonstrate the effectiveness of a new medicine mainly because such a study design provides unbiased estimates of effect. Although primarily used to study efficacy, this study design can also be used to get unbiased estimate of the risk of adverse outcomes. For example, the risk of rare adverse effects with ibuprofen suspension was compared to paracetamol in a randomized trial involving nearly 84,000 children with a febrile illness. No difference was found. Randomization effectively controlled for confounding, including confounding by indication. ${ }^{4}$ Another example of a randomized controlled pharmacoepidemiological study is the Woman's Health Initiative trial conducted between 1993 and 1998 on 16,608 postmenopausal women. This study concluded that estrogen replacement therapy does not prevent development of coronary heart diseases in the postmenopausal women. ${ }^{22}$

RCTs are expensive and due to the tightly controlled environment tend not to reflect a "real world" scenario. Although they remain the gold standard they are used in pharmacoepidemiology mainly for supplementary studies of drug efficacy.

\section{Meta-analysis in pharmacoepidemiology}

Meta-analysis is "the statistical analysis of a collection of analytic results for the purpose of integrating the findings." Metaanalysis of randomized controlled trials is widely accepted as the highest level of evidence for efficacy in health care and is the backbone of evidence-based medicine. ${ }^{23}$ Meta-analysis needs to be used carefully as it is limited by the quality of the original studies (including, for example, design flaws, publication bias and heterogeneity between the studies). It is only the need to make the most efficient use of existing data instead of embarking on a large study that overwhelms the limitations.

In PE, a meta-analysis is especially useful for the study of uncommon adverse reactions, well illustrated by metaanalyses done to confirm the association of NSAIDs use with excess risk of gastrointestinal side effects ${ }^{24}$ or describe the range of relative risks for particular NSAIDs and explore the extent to which differences in toxicity could be related to different doses, or to different susceptibility among patients receiving the various drugs. ${ }^{25}$ Subgroup analysis is also possible with meta-analysis, illustrated well by the paper from Zhou Y et al $(2008)^{26}$ which evaluated the association between green tea consumption and risk of gastric cancer. Individual studies had shown variable results \{the populationbased studies showed a minor inverse association between green tea consumption and risk of gastric cancer ( $\mathrm{OR}=0.68$, $95 \%$ C.I. $[0.49,0.90])$ while no associations were noted with hospital based case control studies (OR $=1.12,95 \%$ C.I. [0.70, $1.77])$ or cohort studies (OR $=1.56,95 \%$ C.I. $[0.93,2.60])\}$. The meta-analysis synthesized data from 14 epidemiological studies, with a total number of 6123 gastric cancer cases and $1,34,006$ controls and the combined results showed that green tea consumption was not associated with the risk of gastric cancer $(\mathrm{OR}=0.98,95 \%$ C.I. $=[0.77,1.24])$. The power to look within subgroups was only available within the context of the meta-analysis, not within any of the individual studies. ${ }^{26}$

Meta-analysis is also useful to assess the effectiveness of existing therapies for new indications, differential effects among subgroups of patients, selection from among several alternative therapies, as well as shortening the time between a medical research finding and the clinical implementation of a new therapy. ${ }^{4}$

\section{Drug utilization studies (DUS)}

Initially PE studies focused on drug safety. However, as awareness that clinical trials conducted during drug 
development were not adequate to generate all information about a new drug, the focus shifted to studying health outcomes of drug use in patients after the drug was marketed. Drug utilization studies (DUS) have therefore become increasingly popular. This concept was developed at the WHO in 1977 to describe investigations into "the marketing, distribution, prescription, and use of drugs in a society, with special emphasis on the resulting medical, social and economic consequences". ${ }^{27}$

DUS are useful to estimate the number of patients exposed to specific drugs within a given time period. The prevalence (all drug users, regardless of when they started to use the drug) or incidence (only patients who started to use the drug within the selected period) can be calculated, the extent of use (including for example use of alternative medicines) at a point in time and/or in a certain area (e.g. in a country, region, community or hospital) can be measured and more importantly used to monitor facilities and even to compare facilities across countries. ${ }^{28}$ DUS can also be used to estimate the extent of rational drug use, and extent of compliance to guidelines. For example, a systematic review of DUS conducted across different levels of hospitals and geographical areas in China found antibiotic utilization to be as high as $50 \%$ in the outpatient setting. ${ }^{29}$ Similar drug utilization studies conducted in India have reported a very high consumption of antibiotics in both public and private sector outpatients, including a high use of broad spectrum and newer antibiotics. ${ }^{30}$ Suitable and sustainable interventions can be designed and implemented to promote rational use of antibiotics based on such DUS to assist in decreasing the menace of antibiotic resistance.

\section{Molecular pharmacoepidemiology}

An area of PE that has greatly developed with burgeoning technological advances has been that of molecular pharmacoepidemiology which focuses on the effects of genetics on clinical outcomes from medication use. While pharmacogenetics is a term predominantly applied to the study of candidate gene variability leading to differences in patients' responses to drugs, the term pharmacogenomics (sometimes used synonymously) refers to a genome-wide approach which simultaneously consider data about thousands of genotypes in drug discovery and development, as well as responses in gene expression to existing medications. Molecular pharmacoepidemiology answers questions related to the population prevalence of single nucleotide polymorphisms (SNPs) and other genetic variants; effects of these SNPs on disease outcomes; what is the impact of gene-drug and gene-gene interactions on disease risk; evaluating the usefulness and impact of genetic tests in populations exposed, or to be exposed, to drugs. ${ }^{4}$

\section{Databases used in pharmacoepidemiological research}

Usually case reports/case series generate hypotheses - in pharmacovigilance programmes these come through spontaneous reporting systems. Ideally to test these hypotheses cohort or case-controlled studies need to be conducted. When the incidence of an adverse effect is 3 per 10,000 or greater cohort or case control studies must include at least 10,000 individuals to be $95 \%$ certain of observing at least one case of any adverse effect. A study of this size is expensive, difficult to perform and may take too long for addressing serious regulatory, commercial, and/or public health crises. Of late therefore computerized databases containing medical care data, the "automated databases," have become popular as potential data sources for pharmacoepidemiology studies.

An ideal database should include inpatient and outpatient records as well those from emergency care and mental health care, all laboratory and radiological tests, and all prescribed and over-the-counter medications, as well as alternative therapies. Further, the database should cover a population large and stable enough to permit discovery of rare events for the drug under study.

Various primary databases like administrative and billing systems used by pharmacies or insurance companies, electronic medical records (more important) and databases for spontaneous reporting system for adverse events are examples of automated databases. The use of databases as primary source of data is a trend that promises to grow in the coming future. ${ }^{4,31}$ Olanzapine and risperidone as a causative agent for the diabetes have been proven by a case control study using general practice research database in United Kingdom. ${ }^{32}$

\section{Pharmacovigilance}

Pharmacoepidemiology developed primarily because of the increasing need to generate data on the safety of a medicine after it had been marketed and Pharmacovigilance programmes use a host of specific methods (apart from those described above, which are used as well) to garner data, all of which are not described in detail here for want of space. These include, for example, Spontaneous reporting, Stimulated reporting, Prescription Event Monitoring, Active surveillance including focused pharmacovigilance and Registries. ${ }^{33,34}$

\section{Pharmacoepidemiology and pharmacokinetics}

The pharmacokinetics (which is the science that describes the time course of the absorption, distribution, metabolism and excretion of drugs within the body) plays a very important role in determining the variability of drug effects. Essentially, pharmacokinetics determines the concentration of drug at its active site, which in turn influences the action of the drug. ${ }^{35}$ Many factors influence the pharmacokinetics of a drug including its physico-chemical properties, dose age, health conditions, concomitant medications, genetic makeup, ${ }^{36}$ all of which will influence the response to the drug. Sometimes, pharmacokinetic studies are conducted to determine whether pharmacokinetic variability can put patients to an increased risk of adverse events. An important goal of pharmacoepidemiology is to use population research methods to characterize factors that influence individual drug response. ${ }^{4}$ Combining pharmacoepidemiology with population pharmacokinetics and the use of pharmacodynamics help in 
determining the magnitude of variability in pharmacokinetics and pharmacodynamics to optimize the therapeutic response to any drug post-marketing.

\section{Role of pharmacoeconomics in pharmacoepidemiology}

Pharmacoeconomics is the branch of health economics concerned with the evaluation of pharmaceutical products in terms of their value for money. Ideally economic evaluations should be incorporated in the clinical trial protocol in all the phases of clinical development. In general, a treatment that costs less but is more effective (and rarely a drug which may cost more but has many clinical benefits) is adopted in treatment plans on the basis of its cost-effectiveness, cost-benefit analysis or favorable incremental cost. Pharmacoeconomics in combination with pharmacoepidemiology can provide a framework to address cost-benefit issues along with risk benefit assessment in drug use policy development. Similarly, pharmacoeconomics could benefit from the robust methods of estimation used in epidemiological research as part of evaluation studies. A closer working relationship between both the disciplines could allow for economic assessment to be made earlier in the product cycle, which could bring benefits to society in terms of making cost-effective products available in the market early and offer them a clear value for money. However, one should keep in mind that pharmacoeconomic analysis is only one of the components of decisionmaking process, which can only substitute the current risk benefit assessment approach. ${ }^{4,37}$

\section{Applications of pharmacoepidemiological (PE) studies}

PE studies are useful to take regulatory decisions and are often required by regulatory agencies to answer specific queries. $\mathrm{PE}$ studies support market penetration by documenting the safety of the drug and protect the drug from "accusations" about adverse effects. They also support marketing strategies to increase name recognition and assist in repositioning the drug (e.g., quality-of-life and economic advantages, effects in special populations, or study of new indications). PE studies are performed in anticipation of future product liability litigation.

\section{Challenges in pharmacoepidemiology and newer approaches}

The major challenge in PE studies is to make the data more rich and robust so that decisions are evidence-based. The need for rapid data access and analysis (for urgent safety issues), statistical power, dealing with bias and confounding, obtaining data from sectors of the health market where currently they are lacking, and consent and confidentiality (protecting the individual but not at the expense of harming the public) form some of the immediate challenges that must be addressed for improving the quality and usefulness of PE studies.
Methods that reduce the probability of confounding by indication and immortal time bias, the two major challenges with PE studies need to be found. Thus, nested case control studies, case- cohort design, within subject designs like case cross over design, case-time control designs and Drug database designs are being developed to meet newer challenges. ${ }^{38}$ Besides these methodological challenges, there are logistic challenges to implement pharmacoepidemiology studies in developing countries. Chief amongst these are underdeveloped spontaneous reporting system, selective reporting of adverse drug reactions due to spurious and counterfeit drugs and lack of automated databases. It is likely that the monitoring of populations with different patterns of morbidity and nutritional status will reveal different types of adverse reactions than what are already known for population in the developed countries even with the already approved drugs. However, challenge lies in implementing pharmacoepidemiological methods in the developing countries which will address the influence of alternative medicines, combined administration of drugs and the unexpected failure of efficacy because of spurious, adulterated or counterfeit medicines. ${ }^{4}$

\section{Future of pharmacoepidemiology}

Pharmacoepidemiology has started to grow and will flourish in future within academia, industry and government. This will be facilitated by methodological advances as well as integration of risk management programs and pharmacogenetics with pharmacoepidemiology. ${ }^{39}$ The areas which need to be explored are drug utilization review, hospital pharmacoepidemiology, pharmacoeconomics, medication adherence, patient safety, and intermediate surrogate markers will empower the decision making process. ${ }^{40}$ Although the future pharmacoepidemiological research will be dominated by automated computer databases, traditional methods will also be important and both can complement each other.

\section{Conflicts of interest}

All authors have none to declare.

\section{R E F E R E N C E S}

1. Stanley K. Design of randomized controlled trials. Circulation. 2007 Mar 6;115(9):1164-1169.

2. Guidance for Industry Good Pharmacovigilance Practices and Pharmacoepidemiologic Assessment available on http:// www.pharmacoepi.org/riskmgmt/fda_pv-pharmacoepi_ 050324.pdf [last accessed on 24.07.13].

3. Hennessy S. Post marketing drug surveillance: an epidemiologic approach. Clin Ther. 1998;20(suppl C):C32-C39.

4. Strom BL, Kimmel SE. Textbook of Pharmacoepidemiology. West Sussex, England: John Wiley \& Sons Ltd; 2006.

5. Roberfroid D, Lachat C, Lucet C. Termination of the CRESCENDO trial. Lancet. 2010 Dec 11;376(9757):1983-1984.

6. Rehman W, Arfons LM, Lazarus HM. The rise, fall and subsequent triumph of thalidomide: lessons learned in drug development. Ther Adv Hematol. 2011 Oct;2(5):291-308. 
7. Rosiglitazone Maleate (marketed as Avandia, Avandamet, and Avandaryl) Information. Available on http://www.fda. gov/drugs/drugsafety/

postmarketdrugsafetyinformationforpatientsandproviders/ ucm143349.htm [Last accessed on 08.01.14].

8. Zhang W, Roederer MW, Chen WQ, Fan L, Zhou HH. Pharmacogenetics of drugs withdrawn from the market. Pharmacogenomics. 2012 Jan;13(2):223-231.

9. Fletcher RH, Fletcher SW, Wagner EH. Clinical epidemiology. The Essentials. 3rd ed. Baltimore: Williams \& Wilkins; 1996.

10. Setia MS. Observational studies: how to go about them? Indian J Dermatol Venereol Leprol. 2008 May-Jun;74(3):288-291.

11. Park K, ed. Principles of Epidemiology and Epidemiologic Methods. Park's Textbook of Preventive and Social Medicine. 22nd ed. India: M/s Banarsidas Bhanot Publishers; 2013:50-127.

12. Song JW, Chung KC. Observational studies: cohort and casecontrol studies. Plast Reconstr Surg. 2010;126(6):2234-2242.

13. Suissa S, Ernst P, Benayoun S, Baltzan M, Cai B. Low-dose inhaled corticosteroids and the prevention of death from asthma. N Engl J Med. 2000 Aug 3;343(5):332-336.

14. Connolly HM, Crary JL, McGoon MD, Hensrud DD, Edwards BS, Edwards WD, Schaff HV. Valvular heart disease associated with fenfluramine-phentermine. N Engl J Med. 1997 Aug 28;337(9):581-588.

15. Stergachis A, Hazlet TK, Boudreaux D. In: DiPiro JT, Talbert RL, Yee GC, Matzke GR, Wells BG, Posey LM, eds. Pharmacoepidemiology Pharmacotherapy a Pathophysiologic Approach. 7th ed. USA: McGraw Hill Inc; 2008:46-55.

16. Szumilas M. J Can Acad Child Adolesc Psychiatr. 2010 August; 19(3):227-229.

17. Weil J, Langman MJ, Wainwright $\mathrm{P}$, et al. Peptic ulcer bleeding: accessory risk factors and interactions with non-steroidal anti-inflammatory drugs. Gut. 2000;46:27-31.

18. Euser AM, Zoccali C, Jager KJ, Dekker FW. Cohort studies: prospective versus retrospective. Nephron Clin Pract. 2009;113(3):c214-c217.

19. Hannaford PC, Iversen L, Macfarlane TV, Elliott AM, Angus V, Lee AJ. Mortality among contraceptive pill users: cohort evidence from Royal College of General Practitioners' oral contraception study. BMJ. 2010 Mar 11;340:c927.

20. Oral Contraceptives and Health: An Interim Report from the Oral Contraception Study of the Royal College of General Practitioners. London: Pitman Medical; 1974.

21. Stampfer MJ, Colditz GA, Willett WC, et al. Postmenopausal estrogen therapy and cardiovascular disease. Ten-year follow-up from the nurses' health study. N Engl J Med. 1991;325(11):756-762.

22. Prentice RL, Langer RD, Stefanick ML, et al. Combined analysis of Women's Health Initiative observational and clinical trial data on postmenopausal hormone treatment and cardiovascular disease. Am J Epidemiol. 2006;163:589-599.

23. Jain V, Sharma R, Singh S. Doing meta-analysis in research: a systematic approach. Indian J Dermatol Venereol Leprol. 2012 May-Jun;78(3):242-250.

24. Chalmers TC, Berrier J, Hewitt P, et al. Meta-analysis of randomized controlled trials as a method of estimating rare complications of non-steroidal anti- inflammatory drug therapy. Aliment Pharmacol Ther. 1988;2(suppl 25):9-26.

25. Henry D, Lim LL, Garcia Rodriguez LA, et al. Variability in risk of gastrointestinal complications with individual non- steroidal anti-inflammatory drugs: results of a collaborative meta-analysis. BMJ. 1996;312:1563-1566.

26. Zhou Y, Li N, Zhuang W, Liu G, et al. Green tea and gastric cancer risk: meta-analysis of epidemiologic studies. Asia Pac J Clin Nutr. 2008;17(1):159-165.

27. World Health Organization, WHO International Working Group for Drug Statistics Methodology, WHO Collaborating Centre for Drug Statistics Methodology, WHO Collaborating Centre for Drug Utilization Research and Clinical Pharmacological Services. Introduction to Drug Utilization Research; 2003. Available at: http://www.whocc.no/filearchive/publications/drug utilization_research.pdf [last accessed 14.10.13].

28. Bhalerao MS, Bolshete PM, Swar BD, et al. Use of and satisfaction with complementary and alternative medicine in four chronic diseases: a cross-sectional study from India. Natl Med J India. 2013 Mar-Apr;26(2):75-78.

29. Yin X, Song F, Gong Y, et al. A systematic review of antibiotic utilization in China. J Antimicrob Chemother. 2013 Nov;68(11):2445-2452.

30. Kotwani A, Holloway K. Trends in antibiotic use among outpatients in New Delhi, India. BMC Infect Dis. 2011 Apr 20;11:99.

31. Takahashi Y, Nishida Y, Asai S. Utilization of health care databases for pharmacoepidemiology. Eur J Clin Pharmacol. 2012;68(2):123-129.

32. Koro CE, Fedder DO, L'Italien GJ, et al. Assessment of independent effect of olanzapine and risperidone on risk of diabetes among patients with schizophrenia: population based nested case-control study. BMJ. 2002 Aug 3;325(7358):243.

33. ICH harmonised tripartite guideline pharmacovigilance planning: E2E available on http://www.ich.org/fileadmin/ Public_Web_Site/ICH_Products/Guidelines/Efficacy/E2E/ Step4/E2E_Guideline.pdf [last accessed on 16.07.13].

34. Mitchell AA, Van Bennekom CM, Louik C. A pregnancyprevention program in women of childbearing age receiving isotretinoin. N Engl J Med. 1995, July 13;333(2):101-106.

35. Brunton LL, Chabner BA, Knollmann BC. Pharmacokinetics: the dynamics of drug absorption, distribution, metabolism and elimination. In: Buxton IL, Benet ZL, eds. Goodman and Gilman's the Pharmacological Basis of Therapeutics. 12th ed. USA: McGraw-Hill Publication; 2011:17-41.

36. Kim YB, Ko MJ, Lee DG, Do JG, Hwang JH. CYP2C9 mutation affecting the individual variability of warfarin dose requirement. Ann Rehabil Med. 2012 Dec;36(6):857-860.

37. Briggs AH, Levy AR. Pharmacoeconomics and pharmacoepidemiology: curious bed fellows or a match made in heaven? Pharmacoeconomics. 2006;24(11):1079-1086.

38. Wacholder S. Practical considerations in choosing between the case-cohort and nested case-control designs. Epidemiology. 1991 Mar;2(2):155-158.

39. Andrews E, Gilsenan A, Cook S. Therapeutic risk management interventions: feasibility and effectiveness. J Am Pharm Assoc. 2004;44(4):491-500.

40. Onder G, Landi F, Cesari M, Gambassi G, Carbonin P, Bernabei R, Investigators of the GIFA Study. Inappropriate medication use among hospitalized older adults in Italy: results from the Italian Group of Pharmacoepidemiology in the Elderly. Eur J Clin Pharmacol. 2003 Jun;59(2):157-162. 